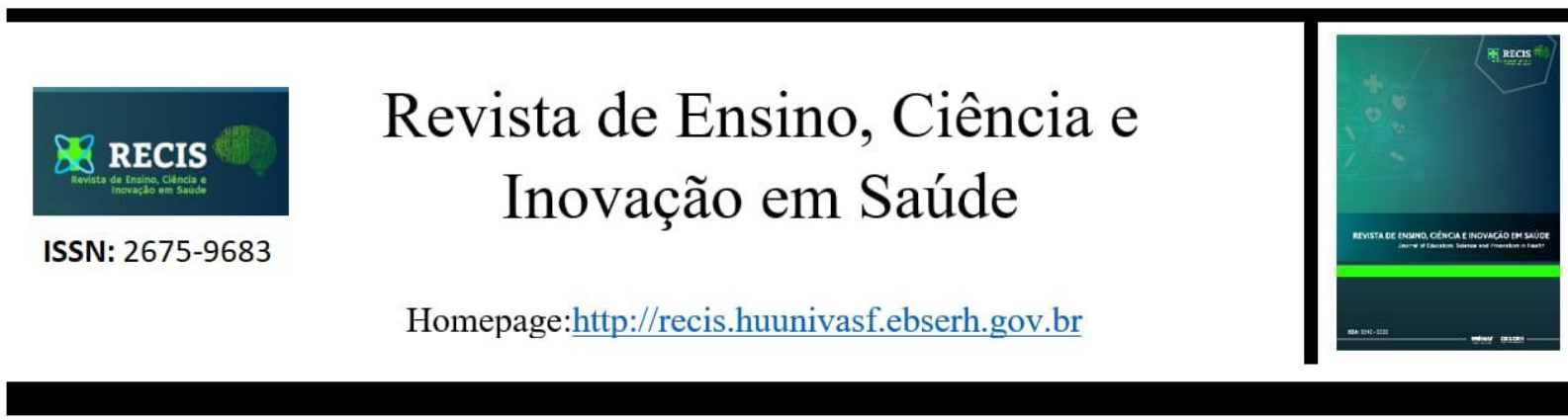

\title{
Levantamento epidemiológico das anomalias congênitas em Minas Gerais entre 2014 e 2018
}

\section{Epidemiological survey of congenital anomalies in Minas Gerais between 2014 and 2018}

\author{
Isadora Caixeta da Silveira Ferreira'; Guilherme Henrique Borges²; Ricardo Ferreira- \\ Nunes ${ }^{3}$. \\ ${ }^{1}$ Universidade Federal de Uberlândia, Instituto de Biologia, Uberlândia, Minas Gerais; ${ }^{2}$ Universidade Federal \\ de Uberlândia, Pós-Graduação em Odontologia, Uberlândia, Minas Gerais; ${ }^{3}$ Instituto de Educação Superior \\ de Brasília, Curso de Farmácia, Brasília, Distrito Federal.
}

Autor correspondente: isadora-biomed@ hotmail.com

Artigo recebido em 23/06/2021 e aceito em 19/07/2021

\begin{abstract}
RESUMO
O objetivo deste estudo foi realizar um levantamento epidemiológico das anomalias congênitas em nascidos vivos em Minas Gerais entre 2014 e 2018. Trata-se de um estudo epidemiológico, descritivo e retrospectivo, realizado com informações secundárias do Sistema de Informações sobre Nascidos Vivos (SINASC). Inicialmente, foi analisado o número total de nascidos vivos. Em seguida, verificou-se: número de casos por ano e tipos de anomalias congênitas em nascidos vivos, bem como sexo, raça/cor, idade gestacional e peso ao nascer. As mães foram analisadas quanto: idade e estado civil, tipo de gravidez e parto. Os dados foram organizados no programa Microsoft Excel@ e foram calculados a frequência $(\mathrm{N})$ e o percentual $(\%)$. As análises estatísticas foram feitas no programa GraphPad Prism® 7. No período analisado, ocorreram 1.310.750 nascimentos em Minas Gerais, dos quais 9.021 apresentaram anomalias congênitas, caracterizando uma prevalência de $0,68 / 100$. Do total de nascidos vivos com anomalias congênitas, 18,90\% foram em 2014 e $21,45 \%$ em 2018, o que representa um aumento de $11,89 \%$. O tipo de anomalia mais frequente foi do aparelho musculoesquelético $(32,41 \%)$. Os mais acometidos foram do sexo masculino $(55,87 \%)$, pardos $(54,38 \%)$, nascidos a termo $(72,74 \%)$, e com peso adequado (70,96\%). Houve predomínio de mães menores de 35 anos $(79,75 \%)$, solteiras $(42,62 \%)$, que tiveram gravidez única $(96,66 \%)$ e parto cesáreo $(63,96 \%)$. Houve aumento da prevalência de anomalias congênitas no período estudado. Essas informações alertam sobre os fatores de risco e podem direcionar ações de saúde pública para melhorar o prognóstico dos acometidos.

Palavras-chave: Anormalidades Congênitas; Epidemiologia; Malformações Congênitas; Prevalência.
\end{abstract}

\section{ABSTRACT}

The aim of this study was to conduct an epidemiological survey of congenital anomalies in live births in Minas Gerais between 2014 and 2018. This is an epidemiological, descriptive, and retrospective study, carried out with secondary information from the Information System on Live Births. (SINASC). Initially, the total number of live births was analyzed. Then, it was verified: the number of cases per year and types of congenital anomalies in live births, as well as gender, race/color, gestational age, and birth weight. Mothers were analyzed regarding age and marital status, type of pregnancy, and delivery. Data were organized in Microsoft Excel ${ }^{\circledR}$ program and frequency $(\mathrm{N})$ and percentage $(\%)$ were calculated. Statistical analyzes were performed using the GraphPad Prism ${ }^{\circledR} 7$ program. In the period analyzed, there were 1,310,750 births in Minas Gerais, of which 9,021 had congenital anomalies, featuring a prevalence of 0.68/100. Of the total number of live births with congenital anomalies, $18.90 \%$ were in 2014 and $21.45 \%$ in 2018, representing an increase of $11.89 \%$. The most frequent type of anomaly was the musculoskeletal system $(32.41 \%)$. The most affected were males

Ferreira ICS, et. al / Revista de Ensino, Ciência e Inovação em Saúde v.2 n.2(2021) p. 31-38 
(55.87\%), brown $(54.38 \%)$, born at term $(72.74 \%)$, and with adequate weight $(70.96 \%)$. There was a predominance of mothers under 35 years old $(79.75 \%)$, single $(42.62 \%)$, who had a single pregnancy $(96,66 \%)$, and cesarean delivery $(63,96 \%)$. There was an increase in the prevalence of congenital anomalies during the study period. This information warns about risk factors and can guide public health actions to improve the prognosis of those affected.

Keywords: Congenital Abnormalities; Epidemiology; Congenital Malformations; Prevalence.

\section{INTRODUÇÃO}

O desenvolvimento embrionário e fetal humano é um período extremamente sensível a diversos fatores genéticos e ambientais. As alterações que surgem no decorrer dessa etapa crítica da vida podem impactar diretamente na saúde, no desenvolvimento e na sobrevivência da criança após o seu nascimento. ${ }^{1}$ As anomalias congênitas, também chamadas de defeitos congênitos ou malformações congênitas, são caracterizadas como alterações estruturais ou funcionais, que se originam ao longo da vida intrauterina e se manifestam no período pós-natal. Elas podem ocorrer de forma isolada por meio de uma única alteração, ou de maneira simultânea através de um conjunto de variações. ${ }^{2}$

Por se tratar de um grupo bastante heterogêneo, as anomalias congênitas apresentam gravidade variável, de acordo com o tipo de manifestação. Aquelas alterações que impactam na expectativa de vida, na saúde e no funcionamento físico e social da criança, podendo inclusive levar ao óbito, são classificadas como anomalias congênitas maiores. ${ }^{2}$ Ao passo que aquelas que estão associadas a um fenótipo sobressalente, porém apresentam um menor impacto na sobrevivência, são denominadas anomalias congênitas menores. Nesse sentido, as anomalias congênitas são um problema de saúde pública, visto que estão associadas a mortalidade neonatal e infantil, bem como a doenças e incapacidades crônicas. E, além de impactarem diretamente na qualidade de vida dos acometidos, também afetam a saúde emocional e financeira dos familiares envolvidos. ${ }^{1}$

Os fatores de risco das anomalias congênitas podem ser classificados como endógenos e exógenos. As causas endógenas são relacionadas a alterações genéticas, como variações cromossômicas ou em um único gene, e a maioria ocorre quando os pais são portadores de alelos que resultam em alguma doença genética ou pode ser resultante de mutações ao acaso. ${ }^{1,3}$ Já as causas exógenas são frequentemente associadas à mãe, como exposição a microrganismos, desnutrição, uso de drogas ilícitas ou medicamentos com ação teratogênica. ${ }^{3}$
Aproximadamente 15 a $25 \%$ das anomalias congênitas são de origem genética e 8 a $12 \%$ são relacionadas a fatores ambientais. Todavia, a maior parte delas, 40 a 60\%, apresenta causa desconhecida. $^{2}$

Estima-se que no mundo, as anomalias congênitas acometam em torno de 2 a $5 \%$ dos nascidos vivos, representando uma carga anual de 5 milhões de nascimentos, dos quais 400.000 são resultantes de falecimentos fetais e 2,5 milhões resultam em óbitos até os cinco anos de idade. ${ }^{3-4} \mathrm{~A}$ maioria dos falecimentos por anomalias congênitas ocorre no primeiro ano de vida. No Brasil, essas anomalias são consideradas a segunda causa de morte infantil mais frequente, sendo responsáveis por aproximadamente $11,2 \%$ dos óbitos nessa faixa etária. ${ }^{2}$ Existe uma grande dificuldade para mensuração precisa da prevalência das anomalias congênitas, visto que muitas mortes fetais ocorrem antes do diagnóstico. ${ }^{5}$

O monitoramento da prevalência e da mortalidade por anomalias congênitas é fundamental, pois alguns fatores como, um bom pré-natal, permitem o diagnóstico precoce, e consequentemente aumentam as chances de sobrevivência. A partir do diagnóstico é realizada a busca por maternidades de referência, o manejo mais adequado para gestação de risco, assim como o aconselhamento genético para as próximas gestações. Além disso, os dados epidemiológicos são fundamentais para pontuar os fatores de risco, tanto maternos quanto neonatais, e assim contribuem com a diminuição da prevalência e mortalidade associada a anomalias decorrentes de causas evitáveis. ${ }^{6}$

No Brasil, uma importante ferramenta para vigilância epidemiológica acerca das anomalias congênitas é o (SINASC), que foi implementado em 1990 e desde 1999 recebe os dados das declarações de nascidos vivos, as quais possuem informações sobre a presença e o tipo de anomalia congênita apresentada pela criança ao nascimento. ${ }^{7}$ Esse sistema possui características singulares que poucos países possuem, como uma vasta cobertura em todo o território nacional e o envio eletrônico de dados. ${ }^{8}$ Apesar de ser um tema relevante, não foram encontrados estudos recentes sobre aspectos epidemiológicos das anomalias congênitas em

Ferreira ICS, et. al / Revista de Ensino, Ciência e Inovação em Saúde v.2 n.2(2021) p. 31-38 
Minas Gerais. Portanto, o objetivo deste estudo foi realizar uma análise epidemiológica a partir do levantamento dos casos de anomalias congênitas em nascidos vivos em Minas Gerais entre 2014 e 2018.

\section{MATERIAL E MÉTODOS}

Este é um estudo epidemiológico, descritivo e retrospectivo, baseado em dados secundários. As informações foram obtidas por meio do levantamento dos casos de anomalias congênitas em nascidos vivos em Minas Gerais, no período de janeiro de 2014 a dezembro de 2018, no SINASC, disponibilizados pelo Departamento de Informática do Sistema Único de Saúde (DATASUS). A coleta das informações foi realizada no mês de outubro de 2020, através do endereço eletrônico (http://www.datasus.com.br). Não foram coletados dados de 2019 e 2020, pois ainda não havia informações suficientes sobre esse período. Por se tratar de informações públicas disponíveis no SINASC/DATASUS, não foi necessária a aprovação pelo Comitê de Ética em Pesquisa com Seres Humanos.

Para calcular a prevalência das anomalias congênitas em nascidos vivos, foram analisadas as variáveis: número de casos de anomalias congênitas em nascidos vivos por ano e número de nascidos vivos por ano em Minas Gerais. A prevalência foi obtida pelo número de casos de anomalias congênitas dividido pelo total de nascidos vivos, multiplicado por 100. Foram obtidas informações sobre os fatores associados ao neonato: sexo, raça ou cor, idade gestacional e peso ao nascer. Também foram coletados dados maternos: idade e estado civil, tipo de gestação e tipo de parto. Por fim, foram averiguados os tipos de anomalias congênitas, de acordo com o Código Internacional de Doenças (CID 10).

Os dados foram plotados e organizados em planilhas no programa Microsoft Excel $^{\circledR}$ versão 2019, em seguida foi feito o cálculo da frequência (N) e do percentual (\%). A análise estatística do número de nascidos vivos com anomalias congênitas por ano foi realizada por meio do teste qui-quadrado, com nível de significância de 5\%. Para facilitar a compreensão dos resultados foram elaborados gráficos e tabelas.

\section{RESULTADOS}

No período analisado, 2014 a 2018, ocorreram 1.310.750 nascimentos em Minas Gerais, sendo 9.021 com anomalias congênitas, caracterizando uma prevalência de 0,68 a cada 100 nascidos vivos. Foram registrados 1.705 nascidos vivos com anomalias congênitas em 2014 (18,90\%), 1.648 em 2015 (18,27\%), 1.819 em 2016 $(20,16 \%), 1.914 \mathrm{em} 2017(21,22 \%)$ e $1.935 \mathrm{em}$ $2018(21,45 \%)$ (Gráfico 1). Houve um aumento estatisticamente significativo no período estudado $\left(\chi^{2}=35,26 ; p<0,05\right)$.

Gráfico 1 - Distribuição do número de nascidos vivos com anomalias congênitas por ano de nascimento em Minas Gerais entre 2014 e 2018.

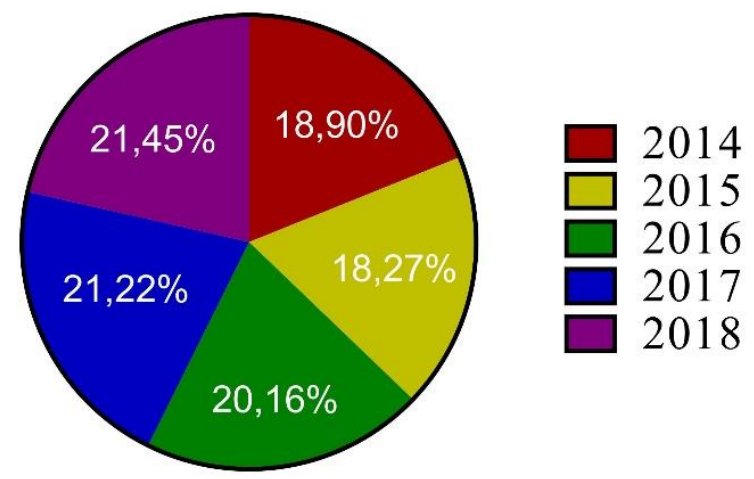

Fonte: Autores, 2021.

Os tipos de anomalias congênitas mais frequentes foram malformações e deformidades congênitas do aparelho osteomuscular (2.921/32,41\%), malformações congênitas nos pés (1.055/11,71\%), malformações congênitas do sistema nervoso $(934 / 10,36 \%)$, malformações congênitas do sistema circulatório $(774 / 8,59 \%)$, malformações congênitas do aparelho geniturinário $(603 / 6,69 \%)$, fenda labial e fenda palatina $(541 / 6,00 \%)$, anomalias cromossômicas (396/4,39\%), malformações congênitas do aparelho digestivo $(331 / 3,67 \%)$, espinha bífida $(301 / 3,34 \%)$, criptorquidia $(102 / 1,13 \%)$, hemangioma e linfangioma (32/0,36\%), ausência, atresia e estenose do intestino delgado $(25 / 0,28 \%)$ e malformações congênitas do quadril $(20 / 0,22 \%)$, respectivamente. Também houveram anomalias enquadradas no grupo outras malformações congênitas (978/10,85\%) (Gráfico 2). 
Gráfico 2 - Distribuição dos nascidos vivos com anomalias congênitas por tipo de anomalia em Minas Gerais entre 2014 e 2018.

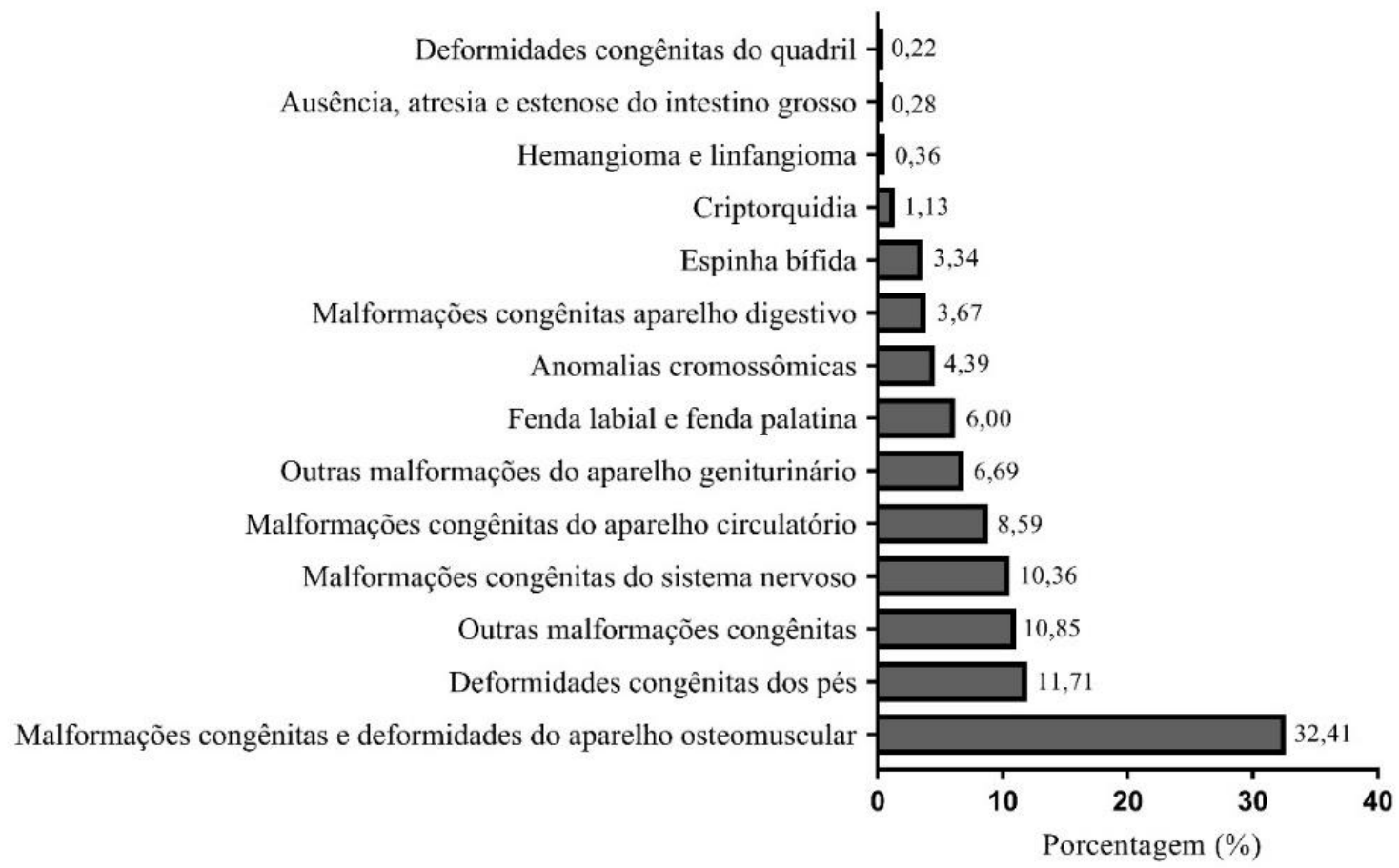

Fonte: Autores, 2021.

No tocante das informações relacionadas ao nascido vivo, observou-se que os mais acometidos por anomalias congênitas eram do sexo masculino (5.040/55,87\%). Houve um predomínio de pardos $(4.906 / 54,38 \%)$, seguidos por brancos $(2.807 / 31,12 \%)$ e negros $(810 / 8,98 \%)$. Um total de $6.365(70,56 \%)$ dos nascidos vivos foram gestados por 37 a 41 semanas (nascimento a termo), e 2.328 $(25,81 \%)$ foram gestados por menos de 36 semanas (nascimento pré-termo, prematuro). Grande parte dos nascidos vivos apresentou peso adequado ao nascer, $2.500 \mathrm{~g}$ ou mais $(6.401 / 70,96 \%)$, todavia $2.617(29,01 \%)$ tiveram baixo peso ao nascer, menos que $2.500 \mathrm{~g}$ (Tabela 1 ).

Por fim, sobre as informações maternas, em $7.194(79,75 \%)$ dos casos de nascidos vivos com anomalias congênitas as mães possuíam menos de 35 anos de idade e em 1.827 (20,35\%) dos casos elas apresentavam 35 anos ou mais (idade materna avançada). Adicionalmente, 3.841 $(42,62 \%)$ mães eram solteiras e $3.722(41,30 \%)$ eram casadas. Sobre o tipo de gestação, a maioria $(8.720 / 96,66 \%)$ era de feto único. Em 5.765 $(63,96 \%)$ nascimentos, o tipo de parto adotado foi a cesárea (Tabela 2).
Tabela 1 - Características epidemiológicas dos nascidos vivos com anomalias congênitas em Minas Gerais entre 2014 e 2018.

\begin{tabular}{lcc}
\hline Variáveis & $\mathbf{N}$ & $\mathbf{\%}$ \\
\hline Sexo & & \\
Masculino & 5.040 & 55,87 \\
Feminino & 3.860 & 42,79 \\
Não informado & 121 & 1,34 \\
Cor ou raça & & \\
Parda & 4.906 & 54,38 \\
Branca & 2.807 & 31,12 \\
Negra & 810 & 8,98 \\
Amarela & 72 & 0,80 \\
Indígena & 25 & 0,28 \\
Não informado & 401 & 4,45 \\
Idade gestacional & & \\
(semanas) & & \\
$<36$ & 2.328 & 25,81 \\
\hline
\end{tabular}

Ferreira ICS, et. al / Revista de Ensino, Ciência e Inovação em Saúde v.2 n.2(2021) p. 31-38 


\begin{tabular}{lcc}
\hline $37-41$ & 6.365 & 70,56 \\
$\geq 42$ & 197 & 2,18 \\
Não informado & 131 & 1,45
\end{tabular}

Peso ao nascer (gramas)

\begin{tabular}{lcc}
$\geq 2.500$ & 6.401 & 70,96 \\
$<2.500$ & 2.617 & 29,01 \\
Não informado & 3 & 0,03 \\
\hline
\end{tabular}

Fonte: Autores, 2021.

Tabela 2 - Características epidemiológicas dos partos e das mães dos nascidos vivos com anomalias congênitas em Minas Gerais entre 2014 e 2018.

\begin{tabular}{ll}
\hline Variáveis & N \\
\hline
\end{tabular}

\section{Faixa etária (anos)}

$\begin{array}{lll}<35 & 7.194 & 79,75 \\ \geq 35 & 1.827 & 20,25\end{array}$

\section{Estado civil}

\begin{tabular}{lcc} 
Solteira & 3.841 & 42,62 \\
Casada & 3.722 & 41,30 \\
Viúva & 21 & 0,23 \\
Separada & 171 & 1,90 \\
Em união consensual & 1.195 & 13,26 \\
Não respondido & 63 & 0,70 \\
Tipo de gestação & & \\
Única & 8.720 & 96,66 \\
Dupla & 280 & 3,10 \\
Tripla ou mais & 4 & 0,04 \\
Não respondido & 17 & 0,19 \\
Tipo de parto & & \\
Vaginal & 3.232 & 35,86 \\
Cesáreo & 5.765 & 63,96 \\
Não respondido & 16 & 0,18 \\
\hline
\end{tabular}

Fonte: Autores, 2021.

\section{DISCUSSÃO}

Este estudo analisou a variação temporal de anomalias congênitas em nascidos vivos no estado de Minas Gerais e identificou alguns fatores associados a ela. Entre 2014 e 2018, a prevalência média de anomalias congênitas foi de 0,68 a cada 100 nascidos vivos, sendo que em 2014 essa prevalência foi de 0,64 e em 2018 foi de 0,74 , o que demonstra um aumento estatisticamente significativo durante $\mathrm{o}$ intervalo analisado $\left(\chi^{2}=35,26 ; p<0,05\right)$. Observou-se que esse valor foi abaixo da média mundial, que é em torno de 2 a $5 \%$ dos nascidos vivos. ${ }^{4}$ A prevalência de anomalias congênitas em nascidos vivos foi 0,92 no Rio Grande do Sul e 1,6 na cidade de São Paulo, contudo em São Luís, capital do Maranhão, essa prevalência foi de $0,49{ }^{9-11}$

Estudos realizados em outros estados do Brasil também apontaram um aumento da prevalência de anomalias congênitas ao longo do tempo, como Bahia, Maranhão e Rio Grande do Sul. ${ }^{9,11-12}$ Até 2015, a prevalência das anomalias congênitas no Brasil era menor que 1\%, embora essas informações estivessem subnotificadas e o número real fosse em torno de 2 a 3\%. Em 2015, com a chegada do Zika vírus, comprovadamente associado a efeitos teratógenos, ocorreu um aumento das notificações de anomalias congênitas, especialmente relacionadas ao sistema nervoso central. Logo, esperava-se que houvesse uma diminuição concomitante do número de casos de Zika vírus e de anomalias congênitas. Entretanto, observou-se que após 2015, houve um aumento na incidência de anomalias congênitas não relacionadas ao sistema nervoso, o que sugere que, possivelmente, a epidemia do Zika vírus estimulou a busca ativa de casos. ${ }^{13}$

A caracterização dos tipos de anomalias congênitas mais comuns em cada região é fundamental para a elaboração de planos de ação e programas para atendimento dos pacientes portadores dessas doenças e orientação de seus familiares. Neste estudo, as malformações e deformidades congênitas do aparelho osteomuscular $(32,41 \%)$ foram as mais frequentes. Em concordância com esses resultados, esse grupo de anomalias congênitas foi associado a 48,04\% dos casos no estado da Bahia, 48,0\% em São Luís e $42,1 \%$ em Recife. ${ }^{11-12,14}$ Acredita-se que a facilidade do diagnóstico desse tipo de anomalia possa contribuir com o diagnóstico precoce e consequentemente com a maior prevalência, visto que elas são caracterizadas como mal formações visíveis ao exame físico. ${ }^{15}$ 
Os resultados encontrados neste estudo mostraram que as anomalias congênitas foram mais frequentes em nascidos vivos do sexo masculino $(55,87 \%)$, pardos $(54,38 \%)$ e brancos $(31,12 \%)$. Com relação ao gênero, esse achado encontra consonância com os dados obtidos em um estudo em Fortaleza, no qual 53\% dos nascidos vivos com anomalias congênitas eram homens, assim como em um estudo em São Luís, em que os homens foram os mais acometidos, representando $54,9 \% .{ }^{11,16}$ Sobre a etnia, de acordo com um estudo realizado nos Estados Unidos, neonatos brancos tiveram um risco maior de malformações craniofaciais, cardíacas e geniturinárias do que negros, contudo esse último grupo apresentou mais chances de apresentar malformações musculoesqueléticas, enquanto no Brasil não foram encontrados estudos sobre essas associações. ${ }^{17}$

Neste estudo, a maioria dos nascidos vivos com anomalias congênitas apresentou $2.500 \mathrm{~g}$ ou mais ao nascer $(70,96 \%)$ e foi gestada por 37 a 41 semanas $(70,56 \%)$. Resultados semelhantes foram observados em Fortaleza, onde 57\% dos neonatos nasceram a termo e $52 \%$ com peso entre $2.500 \mathrm{~g} \mathrm{e}$ 3.999 g. ${ }^{16}$ Outrossim, em São Luís, 83,7\% nasceram a termo e $76,4 \%$ com peso adequado. ${ }^{11}$ Esses achados diferem da literatura, que aponta uma associação entre as anomalias congênitas com a maior ocorrência de parto prematuro, tendo em vista que bebês prematuros podem apresentar até o dobro de anomalias que aqueles nascidos a termo. ${ }^{18}$ Acredita-se que esses resultados revelam que o ganho de peso parece ser mais influenciado pelo tempo de gestação adequado do que pela presença da anomalia congênita.

A correlação entre a idade materna e o risco de anomalias congênitas é uma questão muito abrangente e que ainda não está totalmente elucidada. Sabe-se que a idade materna avançada eleva o risco de anormalidades cromossômicas, sobretudo da síndrome de Down. Entretanto, mães adolescentes também apresentam risco potencializado para outros tipos de anomalias congênitas, como a gastrosquise, que é um defeito no fechamento da parede abdominal. ${ }^{19}$ Neste estudo, em 79,75\% dos casos de nascidos vivos com anomalias congênitas as mães possuíam menos de 35 anos de idade. Ao contrário, no Rio Grande do Sul, a faixa etária com maior frequência de nascidos vivos com anomalias congênitas foi de mães com 40 anos ou mais. ${ }^{9}$ Em ambos os estudos, os tipos mais comuns de anomalias congênitas foram as osteomusculares.
Sobre o tipo de gestação, a maioria $(96,66 \%)$ era de feto único, embora um estudo realizado nos Estados Unidos mostrou que as anomalias congênitas são mais frequentes em gêmeos do que em filhos únicos ${ }^{20}$. Entretanto, em São Luís também foi observado que 97,9\% dos nascidos vivos com anomalias congênitas foram gerados por gestação única. ${ }^{11}$ Em 63,96\% dos nascimentos, o tipo de parto adotado foi a cesárea. Esse achado já era esperado, visto que no Brasil a prevalência de partos por cesárea é bastante elevada, independentemente da situação fetal. Em 2013, 59,32\% dos nascimentos ocorridos na região Sudeste ocorreram por meio de partos cesarianos. ${ }^{21}$

A principal limitação deste estudo foram as falhas ou divergências de informações relacionadas ao preenchimento das declarações de nascidos vivos. Isso ficou evidente, uma vez que houve uma discrepância no número de casos de anomalias congênitas em variáveis distintas analisadas. Quando se observou a quantidade de casos por ano obteve-se o total de 9.021 nascidos vivos com anomalias congênitas no período estudado, contudo na classificação dos tipos de anomalias o total foi de 9.013. Além disso, várias variáveis analisadas tiveram um percentual de respostas ignoradas, o que prejudica a obtenção de resultados fidedignos.

\section{CONCLUSÃO}

A partir dos resultados obtidos, conclui-se que os nascidos vivos mais acometidos por anomalias congênitas em Minas Gerais, entre 2014 e 2018, foram aqueles do sexo masculino, pardos, nascidos a termo e com peso adequado. Outros fatores de risco observados foram: idade materna inferior a 35 anos, gravidez única e parto cesárea. Embora houve um aumento na prevalência no período analisado, acredita-se que ainda existem casos subnotificados no estado. Contudo, a divulgação desses dados epidemiológicos é fundamental para compreensão dos fatores de risco para anomalias congênitas, bem como para direcionar ações de saúde pública com o intuito de reduzir as causas evitáveis e melhorar o aconselhamento genético, os cuidados prestados aos portadores e familiares, e consequentemente a qualidade de vida e o prognóstico dos mesmos.

\section{REFERÊNCIAS}

1. DeSilva M, Munoz FM, Mcmillan M, et al. Congenital anomalies: Case definition and guidelines for data collection, analysis, and presentation of immunization safety data. Vaccine

Ferreira ICS, et. al / Revista de Ensino, Ciência e Inovação em Saúde v.2 n.2(2021) p. 31-38 
2016;34(49):6015.

https://doi.org/10.1016/j.vaccine.2021.01.055

2. Mendes IC, Jesuino RSA, Pinheiro DS, et al. Anomalias congênitas e suas principais causas evitáveis: uma revisão. Revista Médica de Minas Gerais http://www.dx.doi.org/10.5935/2238-

$\underline{3182.20180011}$

3. Modell B, Darlison MW, Malherbe H, et al. Congenital disorders: epidemiological methods for answering calls for action. Journal of Community Genetics 2018;9(4):335. https://doi.org/10.1007/s12687-018-0390-4

4. Feldkamp ML, Carey JC, Byrne JLB, et al. Etiology and clinical presentation of birth defects: population based study. BMJ 2017;357:j2249. https://doi.org/10.1136/bmj.j2249

5. Hanaoka T, Tamura N, Ito K, et al. Prevalence and Risk of Birth Defects Observed in a Prospective Cohort Study: The Hokkaido Study on Environment and Children's Health. Journal of Epidemiology https://doi.org/10.2188/jea.JE20160108

6. Cardoso-dos-Santos AC, Magalhães VS, Medeiros-de-Souza AC, et al. International collaboration networks for the surveillance of congenital anomalies: a narrative review. Epidemiologia e Serviços de Saúde 2020;29(4). https://doi.org/10.5123/S1679-

49742020000400003

7. Geremias AL, Almeida MF de, Flores LPO. Avaliação das declarações de nascido vivo como fonte de informação sobre defeitos congênitos. Revista Brasileira de Epidemiologia 2009;12(1):60-8. https://doi.org/10.1590/S1415790X2009000100007

8. Luquetti DV, Koifman RJ. Quality of birth defect reporting in the Brazilian Information System on Live Births (SINASC): a comparative study of 2004 and 2007. Cadernos de Saúde Pública 2010;26(9):1756-65.

https://doi.org/10.1590/S0102-

311X2010000900009

9. Luz G dos S, Karam S de M, Dumith SC, et al. Anomalias congênitas no estado do Rio Grande do Sul: análise de série temporal. Revista Brasileira de Epidemiologia 2019;22. https://doi.org/10.1590/1980-549720190040 10. Cosme HW, Lima LS, Barbosa LG. Prevalence of congenital anomalies and their associated factors in newborns in the city of São Paulo from 2010 to 2014. Revista Paulista de Pediatria 2017;35(1):33-8. https://doi.org/10.1590/1984$\underline{0462 / ; 2017 ; 35 ; 1 ; 00002}$
11. Rodrigues L dos S, Lima RH da S, Costa LC, et al. Características das crianças nascidas com malformações congênitas no município de São Luís, Maranhão, 2002-2011. Epidemiologia e Serviços de Saúde 2014;23(2):295-304. https://doi.org/10.5123/S1679-

$\underline{49742014000200011}$

12. Andrade A do N, Alves RM, Toralles MBP. Perfil epidemiológico de anomalias congênitas no Estado da Bahia. Revista de Ciências Médicas e Biológicas 2018;17(3):287-91. http://dx.doi.org/10.9771/cmbio.v17i3.28624

13. Paixão ES, Rodrigues MS, Cardim LL, et al. Impact evaluation of Zika epidemic on congenital anomalies registration in Brazil: An interrupted time series analysis. PLOS Neglected Tropical Diseases 2019;13(9):e0007721. https://doi.org/10.1371/journal.pntd.0007721

14. Guimarães ALS, Barbosa CC, Oliveira CM de, et al. Análise das malformações congênitas a partir do relacionamento das bases de dados de nascidos vivos e óbitos infantis. Revista Brasileira de Saúde Materno Infantil 2019;19(4):917-24. https://doi.org/10.1590/180693042019000400010

15. Cosme HW, Lima LS, Barbosa LG, et al. Prevalence of congenital anomalies and their associated factors in newborns in the city of São Paulo from 2010 to 2014. Revista Paulista de Pediatria 2017;35(1):33-8.

https://doi.org/10.1590/1984-

0462/;2017;35;1;00002

16. Fontoura FC, Cardoso MVLML, Fontoura FC, et al. Association between congenital malformation and neonatal and maternal variables in neonatal units of a Northeast Brazilian city. Texto \& Contexto - Enfermagem 2014;23(4):907$14 . \quad$ https://doi.org/10.1590/0104$\underline{07072014002320013}$

17. Egbe A, Lee S, Ho D, et al. Racial/ethnic differences in the birth prevalence of congenital anomalies in the United States. Journal of Perinatal Medicine 2015;43(1):111-7. https://doi.org/10.1515/jpm-2013-0344

18. Jonathan R, Swanson MD, Robert A, et al. Early Births and Congenital Birth Defects: A Complex Interaction. Clinics in Perinatology 2013;40(4):629-44.

https://doi.org/10.1016/j.clp.2013.07.009

19. Souza S de, Nampo FK, Pestana CR. Major birth defects in the Brazilian side of the triple border: a population-based cross-sectional study. Archives of Public Health 2020;78. https://doi.org/10.1186/s13690-020-00443-w 
Revista de Ensino, Ciência e Inovação em Saúde v.2, n. 2 (2021) 31-38

ISSN: 2675-9683/DOI: 10.51909/recis.v2i2.155

20. Dawson AL, Tinker SC, Jamieson DJ, et al. Twinning and major birth defects, National Birth Defects Prevention Study, 1997-2007. Journal of Epidemiology and Community Health 2016;70(11):1114. http://dx.doi.org/10.1136/jech$\underline{\text { 2015-206302 }}$
21. Eufrásio LS, Souza de DE, Fonsêca AMC da, et al. Diferenças regionais brasileiras e fatores associados à prevalência de cesárea. Fisioterapia em Movimento 2018;31. https://doi.org/10.1590/1980-5918.031.AO08 